\title{
The Role of Adjuvant Treatment in Patients with High-Grade Meningioma
}

\author{
Minjae Cho, M.D., Jin-Deok Joo, M.D., In Ah Kim, M.D., Ph.D., ${ }^{2}$ Jung Ho Han, M.D., ${ }^{1}$ Chang Wan Oh, M.D., Ph.D., ${ }^{1}$ \\ Chae-Yong Kim, M.D., Ph.D.' \\ Departments of Neurosurgery, Radiation Oncology, ${ }^{1}$ Seoul National University Bundang Hospital, Seoul National University College of \\ Medicine, Seongnam, Korea
}

Objective : To investigate the efficacy of adjuvant treatment in patients with high-grade meningioma.

Methods : A retrospective analysis was performed for patients with high-grade meningioma, World Health Organization grade 2 or 3, in a single center between 2003 and 2014. The patients were reviewed according to age at diagnosis, sex, the location of meningioma, degree of tumor resection, histological features, and type of adjuvant treatment. These factors were analyzed by Firth logistic regression analyses.

Results : Fifty-three patients with high-grade meningioma were enrolled. Thirty-four patients received adjuvant treatment; conventional radiotherapy or radiosurgery. Clinical follow-up ranged from 13-113 months with a median follow-up of 35.5 months. Gross total removal (GTR), Simpson grade 1 or 2, was achieved in 29 patients and, among them, 13 patients received adjuvant treatment. In the other 24 patients with non-GTR, conventional adjuvant radiotherapy and radiosurgery were performed in 11 and 10 patients, respectively. The other 3 patients did not receive any adjuvant treatment. Radiation-related complications did not occur. Of the 53 patients, 19 patients had suffered from recurrence. The recurrence rate in the adjuvant treatment group was 23.5\% (8 out of 34). On the other hand, the rate for the non-adjuvant treatment group was $57.9 \%$ (11 out of 19 ) (odds ratio $[O R]=0.208, p=0.017$ ). In the GTR group, the recurrence rate was $7.5 \%$ (1 out of 13) for patients with adjuvant treatment and $50 \%$ ( 8 out of 16 ) for patients without adjuvant treatment $(\mathrm{OR}=0.121, p=0.04)$.

Conclusion : Adjuvant treatment appears to be safe and effective, and could lead to a lower recurrence rate in high-grade meningioma, regardless of the extent of removal. Our results might be used as a reference for making decisions when planning adjuvant treatments for patients with high-grade meningioma after surgery.

Key Words : Meningioma · Adjuvant radiotherapy · Radiosurgery.

- Received : November 17, 2016 •Revised : March 1, 2017 •Accepted : April 26, 2017

- Address for reprints : Chae-Yong Kim, M.D., Ph.D.

Department of Neurosurgery, Seoul National University Bundang Hospital, Seoul National University College of Medicine, 82 Gumi-ro 173beon-gil, Bundang-gu, Seongnam 13620, Korea

Tel : +82-31-787-7165, Fax : +82-31-787-4097, E-mail : chaeyong@snu.ac.kr

This is an Open Access article distributed under the terms of the Creative Commons Attribution Non-Commercial License (http://creativecommons.org/licenses/by-nc/4.0) which permits unrestricted non-commercial use, distribution, and reproduction in any medium, provided the original work is properly cited. 


\section{INTRODUCTION}

Meningioma is the most common type of primary intracranial tumor, composing more than 35\% of all ${ }^{3)}$. The World Health Organization's (WHO's) classification of central nervous system tumors, revised in 2007, classifies meningioma into grade 1 (benign), grade 2 (atypical), or grade 3 (malignant or anaplastic). Although the majority are benign, atypical and malignant meningioma, which are termed high-grade meningioma in this study, account for 6-18\% of all meningiomas. The recurrence rates for atypical meningioma and malignant meningioma are known to be approximately $30-40 \%$ and $50-80 \%$, respectively ${ }^{18)}$.

Surgery is the treatment of choice for patients with highgrade meningioma. Due to the high recurrence rate, highgrade meningioma is also commonly treated with adjuvant treatments, such as adjuvant radiotherapy or radiosurgery following surgical removal. It is widely accepted that adjuvant treatments are required for high-grade meningioma that is sub-totally resected (STR). However, the role of adjuvant treatment for high-grade meningioma in patients with gross total removal (GTR) remains controversial, with conflicting reports in previous literature $e^{4,8,14,20)}$.

In this retrospective study, we reviewed the outcomes of high-grade meningioma in 53 patients after surgical removal with or without adjuvant treatments. In particular, this study focused on whether there are benefits to adjuvant treatments in patients with GTR.

\section{MATERIALS AND METHODS}

Our patient database was used to collect patients between 2003 and 2014. All patients were treated with surgical resection and the tumors were pathologically confirmed as either grade 2 or 3 meningioma. All pathologic reports were thoroughly reviewed to confirm that the patients satisfied the definition of grade 2 or 3 meningioma according to the WHO 2000/2007 classification. All patients were included, regardless of the location of the tumor, unless the tumors were located outside the cranium. Among 718 patients undergoing surgery due to newly diagnosed meningioma, 53 (7.3\%) patients with high-grade meningioma were included in this study. The patients were reviewed by age at diagnosis, sex, location of me- ningioma, degree of tumor resection, histological features, and type of adjuvant treatment. Treatment characteristics such as extent of surgical resection, and details of adjuvant treatments were collected for each patient.

\section{Treatment course}

Senior authors (CWO, CYK, and JHH) of Seoul National University Bundang Hospital (SNUBH) performed the surgeries. The tumor resection status was evaluated with Simpson grading and a postoperative magnetic resonance imaging (MRI). Grades 1 and 2 were defined as the GTR of the tumor. Grade 3 and above was defined as non-GTR. Tumor resection was confirmed using an immediate postoperative MRI within 48 hours after surgery, as well as surgical records. Gross total removal was defined as a lack of detectable gadolinium (Gd)enhancing lesions. Early postoperative treatment, termed adjuvant treatment, was defined as radiotherapy or radiosurgery performed before the first 6 months after initial surgery. For radiation therapy, radiation of 54-61.2 Gy was delivered to the postoperative tumor bed during 6 to 7 weeks. For radiosurgery, a Leksell Gamma Knife (GK) PERFEXION ${ }^{\mathrm{TM}}$ (Elekta, Stockholm, Sweden) was used. After acquisition of an axial Gd-enhanced MRI, the lesion was targeted by Leksell Gamma Plan software version 10 (Elekta). The senior authors decided to proceed to the adjuvant treatments in patients with GTR when they had a suspicion that there could be microscopic residual tumor cells although there was no evidence in postoperative MRIs.

\section{Clinical and radiological follow-up}

The first postoperative MRI was followed up 1 or 2 months after surgery. If adjuvant treatments were performed, an MRI was taken 1 month after the treatments. Radiologic studies were then carried out either every 6 or 3 months for grade 2 and grade 3 meningioma, respectively. Gd-enhanced T1weighted sequence was used to evaluate the tumor recurrence and the degree of tumor response to adjuvant treatments. Whether the recurrence is present or not was systematically confirmed by the neuroradiologists in SNUBH. In terms of response, determination of treatment response was performed according to Macdonald criteria, based on the product of orthogonal diameters on the image with the largest Gd-enhanced tumor area ${ }^{16)}$. 


\section{Statistical analysis}

Data were collected using Excel software (Microsoft, Redmond, WA, USA), We tested the normality of the numerical data using a Kolmogorov-Smirnov \& Shapiro-Wilk test. The categorical data was analyzed by using a chi-square test and a Fisher's exact test. The relationship between adjuvant treatment and recurrence rate was analyzed by Firth logistic regression analysis. Progression-free survival points were plotted using Kaplan-Meier estimates. The statistical analysis was performed using STATA version 13 (StataCorp, College Station, TX, USA).

\section{RESULTS}

\section{Baseline patient, tumor, and treatment charac- teristics}

Of the total 53 patients with high-grade meningioma, GTR, Simpson grade 1 or 2 , was achieved in 29 patients. Among these 29 patients, 13 patients received adjuvant radiotherapy. In the other 24 patients with non-GTR, conventional adjuvant radiotherapy and GK were performed in 11 and 10 patients, respectively. The other 3 patients did not receive any adjuvant treatment. For the patients who had received radiotherapy, radiation of 54-61.2 Gy was delivered to postoperative tumor beds during 6 to 7 weeks. Only two patients were given radiation therapy below $54 \mathrm{~Gy}$. One was for the protection of the optic nerve (50 Gy) in the case of tuberculum sella meningioma, and the other was due to an early cessation of radiotherapy (30 Gy) because of increased intracranial pressure after surgery. GK was performed in 10 patients with STR. The dose of radiation ranged from 8.1 to $18 \mathrm{~Gy}$ (mean, $14.2 \mathrm{~Gy}$ ) in a single fraction to the $50 \%$ isodense line, and the target volume ranged from 1.3 to $28.3 \mathrm{~cm}^{3}$ (mean, $11.9 \mathrm{~cm}^{3}$ ).

Baseline patient and tumor characteristics are summarized in Table 1. In the adjuvant treatment group, there are more patients with non-GTR than in the group receiving no adjuvant treatment $(p=0.001)$. Comparison between adjuvant and no adjuvant treatment groups showed no significant difference in age, sex, locations of tumor, size of tumor, and Ki-67 index $(p>0.2)$. There was a trend in which the grade of the meningioma was higher and the number of mitoses per 10 highpower fields (HPFs) was larger in the adjuvant group than in the no adjuvant treatment group, although the trend was not
Table 1. Comparison of patient and tumor characteristics by adjuvant treatment status

\begin{tabular}{|c|c|c|c|}
\hline & AT $(+)$ & AT (-) & $p$-value \\
\hline Total & $34(64.2)$ & $19(35.8)$ & \\
\hline Age & 50 (mean value) & 56 (mean value) & 0.878 \\
\hline \multicolumn{4}{|l|}{ Sex } \\
\hline Male & $18(52.9)$ & $7(36.8)$ & 0.26 \\
\hline Female & $16(47.1)$ & $12(63.2)$ & \\
\hline \multicolumn{4}{|l|}{ Location } \\
\hline Convexity & $8(23.5)$ & $4(21.1)$ & 0.98 \\
\hline Parasagittal & $14(41.2)$ & $7(36.8)$ & \\
\hline Skull base & $7(20.6)$ & $7(36.8)$ & \\
\hline Posterior fossa & $3(8.8)$ & $0(0)$ & \\
\hline Ventricle & $2(5.9)$ & $1(5.3)$ & \\
\hline Tumor size & 5.3 (mean value) & 4.3 (mean value) & 0.98 \\
\hline Ki-67 index* & & & 0.461 \\
\hline \multicolumn{4}{|l|}{ Mitosis (HPFs) } \\
\hline$<5 / 10$ & $12(36.4)$ & $3(15.8)$ & 0.11 \\
\hline$>5 / 10$ & 21 (63.6) & $16(84.2)$ & \\
\hline \multicolumn{4}{|l|}{ Grade } \\
\hline 2 & $25(73.5)$ & $18(94.7)$ & 0.07 \\
\hline 3 & $9(26.5)$ & $1(5.3)$ & \\
\hline \multicolumn{4}{|l|}{ Extent of removal } \\
\hline S1-2 & $13(38.2)$ & $16(84.2)$ & 0.001 \\
\hline S3- & $21(61.8)$ & $3(15.8)$ & \\
\hline
\end{tabular}

Values are presented as number (\%) unless otherwise indicated. *Ki-67 values were not normally distributed numerical data, therefore MannWhitney $\mathrm{U}$ test was used for comparison between adjuvant treatment and non-adjuvant treatment group. AT : adjuvant treatment, HPF : highpower field, S: Simpson grade

statistically significant ( $p=0.11$ and $p=0.07$, respectively). The follow-up period ranged from 13 to 113 months, with a median follow-up period of 35.5 months.

\section{Recurrence rate with or without adjuvant treat- ment, according to the extent of removal}

We decided to perform a multivariate Firth logistic regression test for evaluating the effect of adjuvant treatments in reducing the recurrence rate of high-grade meningioma, with variables having $p$-values lower than 0.2 . The result showed that the recurrence rate was significantly lower in patients who received adjuvant treatments in the GTR group, as well as in total. Of the total 53 patients, 19 patients suffered with re- 
currence. The recurrence rate in the adjuvant treatment group was $23.5 \%$ (8 out of 34 ). On the other hand, the rate for the non-adjuvant treatment group was 57.9\% (11 out of 19) (odds ratio $[\mathrm{OR}]=0.208, p=0.017$ ). Especially, among the 29 patients with GTR, 9 patients had shown recurrence during the follow-up period. The recurrence rate was $7.7 \%$ (1 out of 13 ) for patients with adjuvant treatment and $50 \%$ (8 out of 16) for patients without adjuvant treatment $(\mathrm{OR}=0.121, p=0.04)(\mathrm{Ta}-$ ble 2). All recurrences were in the tumor bed. We plotted a Kaplan-Meier curve for the recurrence-free survival rate, according to the extent of removal (Fig. 1).

\section{Recurrence rate with or without adjuvant treat- ment, within the grade 2 meningioma}

We performed the same logistic regression test within grade
2 meningioma. The result revealed that the patients treated with the adjuvant treatments were statistically less likely to suffer from the recurrence than those without the adjuvant treatments were. While the recurrence rate in the adjuvant treatment group was $28 \%$ (7 out of 25 ), that of the non-adjuvant treatment group was 55.5\% (10 out of 18) (OR=0.147, $p=0.033$ ). The outcome comparison was adjusted for the extent of removal and Mitosis No. per 10 HPFs. However, when we evaluated the recurrence rate within the subgroup in which the patients with GTR and the grade 2 meningioma were included, there was tendency where the recurrence rate was lower in the adjuvant treatment group, but no statistically significant difference, compared with the non-adjuvant treatment group $(\mathrm{OR}=0.163, p=0.118)$ (Table 3$)$.

Table 2. Recurrence rate with or without adjuvant treatment

\begin{tabular}{lccccc}
\hline & AT (-) & AT (+) & Adjusted OR* & 95\% Cl & p-value \\
\hline Total & $11 / 19(57.9)$ & $8 / 34(23.5)$ & 0.208 & $0.057-0.755$ & 0.017 \\
GTR & $8 / 16(50)$ & $1 / 13(7.7)$ & 0.121 & $0.016-0.912$ & 0.040 \\
STR & $3 / 3(100)$ & $7 / 21(33.3)$ & 0.053 & $0.002-1.316$ & 0.073 \\
\hline
\end{tabular}

Values are presented as number (\%). *Outcome comparison was adjusted for grade of MNG and Mitosis No. per 10 HPFs. AT : adjuvant treatment, OR : odds ratio, $\mathrm{Cl}$ : confidence interval, GTR : gross total removal, STR : subtotal removal
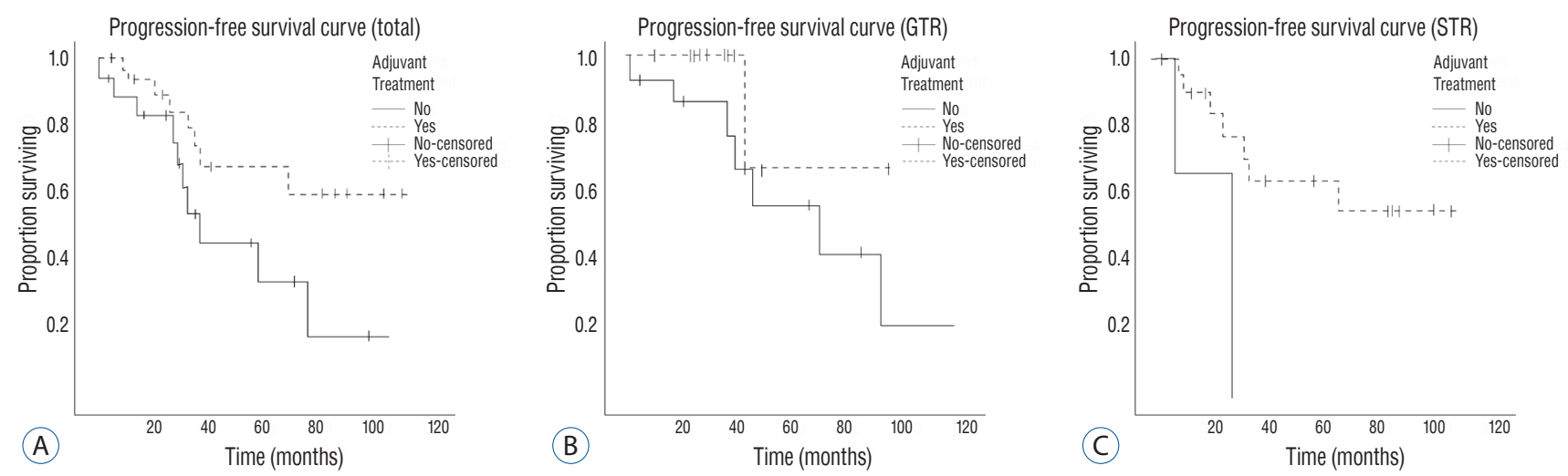

Fig. 1. Progression-free survival curve, according to the extent of removal. A : All patients. B : GTR group. C : STR group. GTR : gross total removal, STR : subtotal removal.

Table 3. Recurrence rate with or without adjuvant treatment within the grade 2 meningioma

\begin{tabular}{lccccc} 
& AT (-) & AT (+) & OR & 95\% Cl & p-value \\
\hline Total & $10 / 18(55.5)$ & $7 / 25(28)$ & $0.147^{*}$ & $0.015-0.457$ & 0.033 \\
GTR & $8 / 16(50)$ & $1 / 13(7.7)$ & $0.163^{\dagger}$ & $0.016-1.675$ & 0.118 \\
\hline
\end{tabular}

Values are presented as number (\%). *Outcome comparison was adjusted for Mitosis No. per 10 HPFs. ${ }^{\dagger}$ Analysis was performed by the Fisher's exact test. AT : adjuvant treatment, $\mathrm{OR}$ : odds ratio, $\mathrm{Cl}$ : confidence interval, GTR : gross total removal 


\section{Complications}

During adjuvant treatment (i.e., radiotherapy and radiosurgery), patients reported fatigue, headache, nausea, dizziness, and skin irritation at portals. However, the degree of the symptoms was not severe, corresponding to grade 2 or below according to Common Toxicity Criteria for Adverse Events (CTCAE) version 4.0. All patients were sufficiently treated with simple medications (e.g., analgesics, steroid, anti-emetics, topical skin agents). According to data, there were no notable long-term complications whose severity was above grade 3 in modified Rankin Score, although there were some reports from patients about memory disturbance, cognitive disturbances, and other non-specific symptoms that are possibly related to radiotherapy.

\section{DISCUSSION}

The aim of this study is to evaluate the efficacy of adjuvant treatments after resection of high-grade meningioma (i.e., grade 2 or 3). In this study, we found that postoperative adjuvant treatments could reduce the recurrence rate in patients in the GTR group.

Although it is maximal surgical removal for all meningioma, irrespective of the grade, that most affects the progno$\operatorname{sis}^{4,13,17,19)}$, high-grade meningioma is well known to recur even after GTR. If it recurs, repeated surgical procedures usually ensue. This poses problems related not only to mortality but also to morbidity, significantly reducing the quality of life ${ }^{15)}$. Therefore, reducing the recurrence rate is closely associated with better outcomes. However, the literature that has been published during the last 10 years has given controversial results about the role of adjuvant radiotherapy after GTR. According to our results, this study gives more strength to the policy that recommends routine postoperative radiotherapy even after GTR.

In 2009, Aghi et al. ${ }^{1)}$ found a high rate of recurrence (41\%) for atypical meningioma 5 years after GTR; although their data did not show a statistically significant effect, the authors found a suggested benefit of postoperative radiation in tumors after GTR. In 2012, Komotar et al. ${ }^{9)}$ included only a GTR group in their study and presented that there was no recurrence in $92 \%$ of patients who received postoperative radiotherapy compared with $59 \%$ of patients who did not $(p=0.085)$, demonstrating a strong trend towards improved local controls with adjuvant radiotherapy. In contrast, there are still many papers arguing against routine postoperative radiotherapy after GTR. In a 2011 study evaluating the role of postoperative radiotherapy for 114 patients after resection for atypical meningioma, Mair et al. ${ }^{12)}$ found that neither radiotherapy nor the extent of resection statistically impacted recurrence for the entire group; these authors then recommended against postoperative radiotherapy for patients after GTR of their tumors. In 2013, Lee et al. ${ }^{11)}$ reported that although radiotherapy significantly decreased the risk of recurrence among patients after STR, there was no statistical effect after GTR. In the same year, Hardesty et al. ${ }^{6}$ suggested that neither adjuvant radiotherapy nor adjuvant radiosurgery affected the tumor recurrence rate.

However, according to a systematic review article published in 2014, the most important cause of these disparate results driven from the previous studies is a consequence of flaws in each paper. This study has reviewed a total of 14 studies published from 1994 to 2011. They insisted that several studies showed trends toward clinical benefits with adjuvant radiotherapy and that the paucity of statistical significance is likely a result of small sample sizes and a lack of statistically significant correlation ${ }^{8)}$. Furthermore, they found that an increased radiation dose almost always correlated with better prognosis in terms of disease control, which makes it probable that adjuvant radiotherapy plays a positive role compared with surgery alone. Additionally, in 2015, Hasan et al. ${ }^{7}$, through a metaanalysis, conclude that adjuvant radiotherapy provides improved local control of atypical meningioma. This study includes the patients from most of the articles mentioned above and has shown the overall benefit of the adjuvant radiotherapy for reducing the recurrence rate, regardless of the conclusions derived from each paper.

In our study, relatively constant intervals between surgery, adjuvant treatments and routine imaging follow-up helped to better assess the relationship between the adjuvant treatments and the recurrence rate. All patients in the adjuvant treatment group had received the treatment within 6 months after the first surgery. It has been reported that adjuvant radiotherapy seems to be more likely to control a disease when it is administered during the initial diagnosis rather than during the recurrence of high-grade meningioma ${ }^{8)}$. Dziuk et al. ${ }^{5)}$ found that adjuvant radiotherapy at initial diagnosis was associated with 
a reduced recurrence rate of malignant meningioma (20\% vs. $67 \%$ ), while patients with recurrent disease fared poorly regardless of adjuvant radiotherapy administration (75\% radiotherapy-treated vs. 78\% untreated). After surgery, the first postoperative MRI was followed up 1 month after surgery. Then, almost all patients visited every 3 or 6 months for their MRI follow-up. In most other retrospective studies, the authors perform a time-to-event analysis, depending on patients' symptoms, to decide whether or not to follow-up with an imaging study.

The adverse effects of radiotherapy on the brain should always be taken into account when patients are given this treatment. It is important to consider these effects thoroughly before making a consensus to support routine adjuvant radiotherapy, regardless of the extent of removal. The adverse effects include radionecrosis, malignant transformations, and delayed cognitive disorders. However, these sequelae may become less problematic with the introduction of more modern methods of radiation delivery, such as intensity-modulated radiotherapy, and reduced radiation exposure to adjacent brain/ neural structures ${ }^{10)}$. Furthermore, van Nieuwenhuizen et al. ${ }^{21)}$ demonstrated that radiotherapy following surgery did not have additional deleterious effects on impaired long-term neurocognitive function in meningioma patients, and it was suggested that neurocognitive deficits could be partly attributed to the use of antiepileptic drugs as well as tumor location, but not to the use of radiotherapy ${ }^{2}$.

The current study has several limitations including the retrospective nature of the study, the relatively small number of patients, and the possible heterogeneous decisions about adjuvant treatment according to the physicians even within the same institute. Additionally, the patient group was not homogeneous in that both grade 2 and 3 meningioma are grouped together and classified as high-grade meningioma. Although we indeed showed the efficacy of the adjuvant treatment in reducing the recurrence within grade 2 meningioma, we could not show the same efficacy in the most controversial group which the patients with grade 2 meningioma and GTR belong to. This is because the number of the patients was insufficient to reveal a statistically significant conclusion. Further validation will be possible through the results from the randomized trials with large numbers of patients.

\section{CONCLUSION}

This study showed that adjuvant treatment appears to be safe and effective, and could lead to a lower recurrence rate in high-grade meningioma, regardless of the extent of removal. Our results might be used as a reference for decision making when planning adjuvant treatment for patients with highgrade meningioma after surgery.

\section{References}

1. Aghi MK, Carter BS, Cosgrove GR, Ojemann RG, Amin-Hanjani S, Martuza $\mathrm{RL}$, et al. : Long-term recurrence rates of atypical meningiomas after gross total resection with or without postoperative adjuvant radiation. Neurosurgery 64 : 56-60; discussion 60, 2009

2. Dijkstra $M$, van Nieuwenhuizen $D$, Stalpers LJ, Wumkes $M$, Waagemans $M$, Vandertop WP, et al. : Late neurocognitive sequelae in patients with WHO grade I meningioma. J Neurol Neurosurg Psychiatry 80 : 910-915, 2009

3. Dolecek TA, Propp JM, Stroup NE, Kruchko C : CBTRUS statistical report: primary brain and central nervous system tumors diagnosed in the United States in 2005-2009. Neuro Oncol 14 Suppl 5 : v1-v49, 2012

4. Durand $A$, Labrousse $F$, Jouvet $A$, Bauchet $L$, Kalamaridès $M$, Menei $P$, et al. : WHO grade II and III meningiomas: a study of prognostic factors. J Neurooncol 95 : 367-375, 2009

5. Dziuk TW, Woo S, Butler EB, Thornby J, Grossman R, Dennis WS, et al. Malignant meningioma: an indication for initial aggressive surgery and adjuvant radiotherapy. J Neurooncol 37 : 177-188, 1998

6. Hardesty DA, Wolf AB, Brachman DG, McBride HL, Youssef E, Nakaji $P$, et al. : The impact of adjuvant stereotactic radiosurgery on atypical meningioma recurrence following aggressive microsurgical resection.

J Neurosurg $119:$ 475-481, 2013

7. Hasan S, Young M, Albert T, Shah AH, Okoye C, Bregy A, et al. : The role of adjuvant radiotherapy after gross total resection of atypical meningiomas. World Neurosurg $83: 808-815,2015$

8. Kaur G, Sayegh ET, Larson A, Bloch O, Madden M, Sun MZ, et al. : Adjuvant radiotherapy for atypical and malignant meningiomas: a systematic review. Neuro Oncol 16 : 628-636, 2014

9. Komotar RJ, lorgulescu JB, Raper DMS, Holland EC, Beal K, Bilsky MH, et al. : The role of radiotherapy following gross-total resection of atypical meningiomas. J Neurosurg 117 : 679-686, 2012

10. Lawrence YR, Li XA, el Naqa I, Hahn CA, Marks LB, Merchant TE, et al. : Radiation dose-volume effects in the brain. Int J Radiat Oncol Biol Phys 76(3 Suppl) : S20-S27, 2010

11. Lee KD, DePowell JJ, Air EL, Dwivedi AK, Kendler A, McPherson CM : Atypical meningiomas: is postoperative radiotherapy indicated? Neurosurg Focus 35 : E15, 2013

12. Mair R, Morris K, Scott I, Carroll TA : Radiotherapy for atypical menin- 
Adjuvant Treatment in High-Grade Meningioma | Cho M, et al.

giomas. J Neurosurg $115: 811-819,2011$

13. Palma L, Celli P, Franco C, Cervoni L, Cantore G : Long-term prognosis for atypical and malignant meningiomas: a study of 71 surgical cases. J Neurosurg 86 : 793-800, 1997

14. Park HJ, Kang HC, Kim IH, Park SH, Kim DG, Park CK, et al. : The role of adjuvant radiotherapy in atypical meningioma. J Neurooncol 115 : 241-247, 2013

15. Poon MT, Fung LH, Pu JK, Leung GK : Outcome comparison between younger and older patients undergoing intracranial meningioma resections. J Neurooncol $114:$ 219-227, 2013

16. Provenzale JM, Ison C, DeLong D : Bidimensional measurements in brain tumors: assessment of interobserver variability. AJR Am J Roentgenol 193 : W515-W522, 2009

17. Sanai N, Sughrue ME, Shangari G, Chung K, Berger MS, McDermott MW :
Risk profile associated with convexity meningioma resection in the modern neurosurgical era. J Neurosurg 112 : 913-919, 2010

18. Saraf S, McCarthy BJ, Villano JL : Update on meningiomas. Oncologist $16: 1604-1613,2011$

19. Simpson $D$ : The recurrence of intracranial meningiomas after surgical treatment. J Neurol Neurosurg Psychiatry 20 : 22-39, 1957

20. Stessin AM, Schwartz A, Judanin G, Pannullo SC, Boockvar JA, Schwartz $T H$, et al. : Does adjuvant external-beam radiotherapy improve outcomes for nonbenign meningiomas? A Surveillance, Epidemiology, and End Results (SEER)-based analysis. J Neurosurg 117 : 669-675, 2012

21. van Nieuwenhuizen D, Klein M, Stalpers LJA, Leenstra S, Heimans JJ, Reijneveld JC : Differential effect of surgery and radiotherapy on neurocognitive functioning and health-related quality of life in WHO grade I meningioma patients. J Neurooncol 84 : 271-278, 2007 